\title{
IDEALIZATION AND POLYNOMIAL IDENTITIES
}

\author{
Malik Bataineh and D. D. Anderson \\ Jordan University of Science and Technology, Jordan and The University of \\ Iowa, USA
}

\begin{abstract}
Let $R$ be a commutative ring, let $M$ be an $R$-module, let $f\left(X_{1}, \ldots, X_{n}\right)$ be a polynomial (with coefficients from $R$ or $\mathbb{Z}$ ) and let $k$ be a positive integer. We show that if $R$ satisfies the polynomial identity

$$
\prod_{i=1}^{k} f\left(X_{1 i}, \ldots, X_{n i}\right)=0,
$$
\end{abstract}

then the idealization $R(+) M$ satisfies

$$
\prod_{i=1}^{k+1} f\left(X_{1 i}, \ldots, X_{n i}\right)=0 .
$$

\section{INTRODUCTION}

Throughout all rings will be commutative, but not necessarily with identity. For rings with an identity, all modules are assumed to be unital. When considering polynomials over a ring, it is useful for the ring to have an identity. So we define the ring $R^{1}$ as follows. If $R$ has an identity, $R^{1}=R$. If $R$ does not have an identity, let $R^{1}$ be the Dorroh extension of $R$ with char $R^{1}=\operatorname{char} R$ (so $R^{1}=R \oplus \mathbb{Z}$ if $\operatorname{char} R=0$ while $R^{1}=R \oplus \mathbb{Z}_{n}$ if char $R=n>0$ with product $\left.\left(r_{1}, n_{1}\right)\left(r_{2}, n_{2}\right)=\left(r_{1} r_{2}+n_{2} r_{1}+n_{1} r_{2}, n_{1} n_{2}\right)\right)$. Suppose that $R$ and $S$ are rings so that $S$ is an $R$-module and $n$ is a positive integer. Recall that for a polynomial $f \in R\left[X_{1}, \ldots, X_{n}\right]$ we say that $S$ satisfies a polynomial identity $f\left(X_{1}, \ldots, X_{n}\right)=0$ if $f\left(s_{1}, \ldots, s_{n}\right)=0$ for any $s_{1}, \ldots, s_{n} \in S$.

Let $R$ be a ring and $M$ an $R$-module. The idealization or trivial extension $R(+) M$ of $R$ and $M$ is the ring with additive group $R \oplus M$ and multiplication

2010 Mathematics Subject Classification. 13B25.

Key words and phrases. Idealization, trivial extension, polynomial identity. 
48

M. BATAINEH AND D. D. ANDERSON

given by $\left(r_{1}, m_{1}\right)\left(r_{2}, m_{2}\right)=\left(r_{1} r_{2}, r_{1} m_{2}+r_{2} m_{1}\right)$. Here $(0 \oplus M)^{2}=0$, so the nilradical of $R(+) M$ is $\operatorname{nil}(R(+) M)=\operatorname{nil}(R) \oplus M$. For results on idealization, the reader is referred to [2].

Now let $R$ be a ring, $M$ an $R$-module and let $k$ be a positive integer. The main result of this note (Theorem 2.2) is the following. If there is a polynomial $f$ in $n$ variables so that $R$ satisfies a polynomial identity

$$
f\left(X_{11}, \ldots, X_{n 1}\right) \cdots f\left(X_{1 k}, \ldots, X_{n k}\right)=0
$$

(resp., $\left(f\left(X_{1}, \ldots, X_{n}\right)\right)^{k}=0$ ), then the idealization $R(+) M$ of $R$ and $M$ satisfies the polynomial identity

$$
f\left(X_{11}, \ldots, X_{n 1}\right) \cdots f\left(X_{1 k}, \ldots, X_{n k}\right) f\left(X_{1 k+1}, \ldots, X_{n k+1}\right)=0
$$

(resp., $\left.\left(f\left(X_{1}, \ldots, X_{n}\right)\right)^{k+1}=0\right)$. In Section 3 we give a number of applications of our result and examples to show the sharpness of the result.

2. Main Result

Now let $\left(r_{1}, m_{1}\right), \ldots,\left(r_{n}, m_{n}\right) \in R(+) M$. For later use first observe that

$$
\prod_{i=1}^{n}\left(r_{i}, m_{i}\right)=\left(\prod_{i=1}^{n} r_{i}, \sum_{i=1}^{n}\left(\prod_{\substack{j=1 \\ j \neq i}}^{n} r_{j}\right) m_{i}\right) .
$$

Proposition 2.1. Let $R$ be $a$ ring and $M$ an $R$-module. Let $f$ be a polynomial and elements $\left(r_{1}, m_{1}\right), \ldots,\left(r_{n}, m_{n}\right)$ be as above. Then

$$
f\left(\left(r_{1}, m_{1}\right), \ldots,\left(r_{n}, m_{n}\right)\right)=\left(f\left(r_{1}, \ldots, r_{n}\right), \sum_{i=1}^{n} \frac{\partial f}{\partial X_{i}}\left(r_{1}, \ldots, r_{n}\right) m_{i}\right) .
$$

In particular, for the case $n=1$ we have

$$
f(r, m)=\left(f(r), f^{\prime}(r) m\right) .
$$

ProOF. Writing $f$ as a linear combination of primitive polynomials, it suffices to prove the result for $f\left(X_{1}, \ldots, X_{n}\right)=X_{1}^{s_{1}} \cdots X_{n}^{s_{n}}$. Now for $r \in R$ and $m \in M$, it is easily proved by induction that $(r, m)^{k}=\left(r^{k}, k r^{k-1} m\right)$. Hence

$$
\begin{aligned}
f\left(\left(r_{1}, m_{1}\right), \ldots,\left(r_{n}, m_{n}\right)\right) & =\left(r_{1}, m_{1}\right)^{s_{1}} \cdots\left(r_{n}, m_{n}\right)^{s_{n}} \\
& =\left(r_{1}^{s_{1}}, s_{1} r_{1}^{s_{1}-1} m_{1}\right) \cdots\left(r_{n}^{s_{n}}, s_{n} r_{n}^{s_{n}-1} m_{n}\right) \\
& =\left(r_{1}^{s_{1}} \cdots r_{n}^{s_{n}}, \sum_{i=1}^{n} s_{i} r_{1}^{s_{1}} \cdots r_{i-1}^{s_{i}-1} r_{i}^{s_{i}-1} r_{i+1}^{s_{i+1}} \cdots r_{n}^{s_{n}} m_{i}\right) \\
& =\left(f\left(r_{1}, \ldots, r_{n}\right), \sum_{i=1}^{n} \frac{\partial f}{\partial X_{i}}\left(r_{1}, \ldots, r_{n}\right) m_{i}\right) .
\end{aligned}
$$


Now we give our main result.

Theorem 2.2. Let $R$ be a ring and $M$ an $R$-module. Let $f=$ $f\left(X_{1}, \ldots, X_{n}\right) \in R^{1}\left[X_{1}, \ldots, X_{n}\right]$ or $f \in \mathbb{Z}\left[X_{1}, \ldots, X_{n}\right]$ and let $k$ be a positive integer. Suppose that $R$ satisfies the polynomial identity $\prod_{i=1}^{k} f\left(X_{1 i}, \ldots, X_{n i}\right)$ $=0$ (resp., $\left.\left(f\left(X_{1}, \ldots, X_{n}\right)\right)^{k}=0\right)$. Then $R(+) M$ satisfies the polynomial identity $\prod_{i=1}^{k+1} f\left(X_{1 i}, \ldots, X_{n i}\right)=0\left(\right.$ resp.,$\left.\left(f\left(X_{1}, \ldots, X_{n}\right)\right)^{k+1}=0\right)$.

Proof. We are given that $\prod_{i=1}^{k} f\left(r_{1 i}, \ldots, r_{n i}\right)=0$ for any $\left\{r_{i j}\right\}_{\substack{1 \leq i \leq n \\ 1 \leq j \leq k}} \subseteq$ $R$ (resp., $\left(f\left(r_{1}, \ldots, r_{n}\right)\right)^{k}=0$ for any $\left.r_{1}, \ldots, r_{n} \in R\right)$. We need that

$$
\prod_{i=1}^{k+1} f\left(\left(r_{1 i}, m_{1 i}\right), \ldots,\left(r_{n i}, m_{n i}\right)\right)=0 \text { for any }\left\{\left(r_{i j}, m_{i j}\right)\right\} \underset{\substack{1 \leq i \leq n \\ 1 \leq j \leq k+1}}{\subseteq} \subseteq R(+) M
$$

(resp., $\left(f\left(\left(r_{1}, m_{1}\right), \ldots,\left(r_{n}, m_{n}\right)\right)^{k+1}=0\right.$ for any $\left(r_{1}, m_{1}\right), \ldots,\left(r_{n}, m_{n}\right) \in$ $R(+) M))$.

We prove the first statement; for the second one we proceed in a similar way. Using Proposition 2.1 and the displayed equality before it we get that

$$
\begin{aligned}
& \prod_{i=1}^{k+1} f\left(\left(r_{1 i}, m_{1 i}\right), \ldots,\left(r_{n i}, m_{n i}\right)\right) \\
& \quad=\prod_{i=1}^{k+1}\left(f\left(r_{1 i}, \ldots, r_{n i}\right), \sum_{j=1}^{n} \frac{\partial f}{\partial X_{j}}\left(r_{1 i}, \ldots, r_{n i}\right) m_{j i}\right) \\
& \quad=\left(\prod_{i=1}^{k+1} f\left(r_{1 i}, \ldots, r_{n i}\right), \sum_{i=1}^{k+1}\left(\prod_{\substack{j=1 \\
j \neq i}}^{k+1} f\left(r_{1 j}, \ldots, r_{n j}\right)\right) \sum_{j=1}^{n} \frac{\partial f}{\partial X_{j}}\left(r_{1 i}, \ldots, r_{n i}\right) m_{j i}\right) \\
& =(0,0)
\end{aligned}
$$

for the last equality use that

$$
\prod_{j=1}^{k+1} f\left(r_{1 i j}, \ldots, r_{n i}\right)=\prod_{\substack{\ell=1 \\ \ell \neq i}}^{k+1} f\left(r_{1 \ell}, \ldots, r_{n \ell}\right)=0 .
$$

\section{EXAMPLES}

The purpose of this section is to illustrate the sharpness of Theorem 2.2 and to illustrate Theorem 2.2 for certain simple polynomial identities. We begin by noting a dichotomy in the case of whether $R$ is reduced.

Suppose that $R$ is reduced, i.e., $\operatorname{nil}(R)=0$. If $R$ satisfies $\prod_{i=1}^{k} f\left(X_{1 i}, \ldots\right.$, $\left.X_{n i}\right)=0\left(\right.$ or $\left.\left(f\left(X_{1}, \ldots, X_{n}\right)\right)^{k}=0\right)$, then $R$ satisfies $\left(f\left(X_{1}, \ldots, X_{n}\right)\right)^{k}=0$ and hence $f\left(X_{1}, \ldots, X_{n}\right)=0$ because $R$ is reduced. Hence if $R$ satisfies 
$\prod_{i=1}^{k} f\left(X_{1 i}, \ldots, X_{n i}\right)=0$ or $\left(f\left(X_{1}, \ldots, X_{n}\right)\right)^{k}=0$ for some $k \geq 1$, then $R(+) M$ satisfies $\prod_{i=1}^{k} f\left(X_{1 i}, \ldots, X_{n i}\right)=0$.

However, if $R$ is not reduced, $R$ can satisfy $\left(f\left(X_{1}, \ldots, X_{n}\right)\right)^{2}=0$ but not $\prod_{i=1}^{2} f\left(X_{1 i}, \ldots, X_{n i}\right)=0$ as our first example shows.

EXAmple $3.1\left(R\right.$ satisfies $(f(X))^{2}=0$ but not $\left.f(X) f(Y)=0\right)$. Take $R$ to be the maximal ideal $(x, y)=(X, Y) /\left(X^{2}, Y^{2}\right)$ of the local ring $\mathbb{Z}_{2}[x, y]=$ $\mathbb{Z}_{2}[X, Y] /\left(X^{2}, Y^{2}\right)$, and $f(X)=X$. Then $(f(r))^{2}=r^{2}=0$ for each $r \in R$, but $f(x) f(y)=x y \neq 0$. For an example where the ring has an identity we may take $R=\mathbb{Z}_{4}(+) \mathbb{Z}_{4}$, and $f(X)=X(1+X)$. It is easily checked that $R$ satisfies $(f(X))^{2}=0$ but does not satisfy $f(X) f(Y)=0$.

We next illustrate the sharpness of Theorem 2.2. Suppose that the ring $R$ satisfies $\prod_{i=1}^{k} f\left(X_{1 i}, \ldots, X_{n i}\right)=0$, but not $\prod_{i=1}^{k-1} f\left(X_{1 i}, \ldots, X_{n i}\right)=0$. So for a nonzero $R$-module $M, R(+) M$ satisfies $\prod_{i=1}^{k+1} f\left(X_{1 i}, \ldots, X_{n i}\right)=$ 0 . We next give examples to show that $R(+) M$ may or may not satisfy $\prod_{i=1}^{k} f\left(X_{1 i}, \ldots, X_{n i}\right)=0$.

EXAmple $3.2\left(R\right.$ satisfies $\prod_{i=1}^{k} f\left(X_{i}\right)=0, R(+) M$ satisfies $\prod_{i=1}^{k+1} f\left(X_{i}\right)$ $=0$, but $R(+) M$ does not satisfy $\left.\prod_{i=1}^{k} f\left(X_{i}\right)=0\right)$. Let $f(X)=X(1+X)$ and $f_{k}=f\left(X_{1}\right) \cdots f\left(X_{k}\right)$. Put $R_{1}=\mathbb{Z}_{2}$ and for $k \geq 1, R_{k+1}=R_{k}(+) R_{k}$. Now $R_{1}$ satisfies $f_{1}=0$ so $R_{k}$ satisfies $f_{k}=0$. It is easily checked that $R_{2}$ does not satisfy $f_{1}=0$. Suppose that $R_{k}$ does not satisfy $f_{k-1}=0$. So there exist $r_{1}, \ldots, r_{k-1} \in R_{k}$ with $r_{1} \cdots r_{k-1}\left(1+r_{1}\right) \cdots\left(1+r_{k-1}\right) \neq 0$. Consider $\left(r_{1}, 0\right), \ldots,\left(r_{k-1}, 0\right),(0,1) \in R_{k}(+) R_{k}=R_{k+1}$. Then

$$
\begin{aligned}
& \left(r_{1}, 0\right) \cdots\left(r_{k-1}, 0\right)(0,1)\left((1,0)+\left(r_{1}, 0\right)\right) \cdots\left((1,0)+\left(r_{k-1}, 0\right)\right)((1,0)+(0,1)) \\
& =\left(r_{1} \cdots r_{k-1}\left(1+r_{1}\right) \cdots\left(1+r_{k-1}\right), 0\right)(0,1)(1,1) \\
& =\left(0, r_{1} \cdots r_{k-1}\left(1+r_{1}\right) \cdots\left(1+r_{k-1}\right)\right) \neq(0,0) .
\end{aligned}
$$

So $R_{k+1}$ does not satisfy $f_{k}$.

$(R$ and $R(+) M$ satisfy $f=0)$. Let $R=\mathbb{Z}_{4}, M=\mathbb{Z}_{2}$ and $f(X)=$ $X^{2}(1+X)^{2}$. Then both $\mathbb{Z}_{4}$ and $\mathbb{Z}_{4}(+) \mathbb{Z}_{2}$ satisfy $f(X)=0$.

Now we would like to illustrate Theorem 2.2 with some simple polynomial identities. But first observe that if $R$ satisfies a polynomial identity $f\left(X_{1}, \ldots, X_{n}\right)=0$, then $f$ must necessarily have the constant term 0 . The interpretation of our theorem for the simplest cases $f(X)=X$ and $f(X)=r X, r \in R$, are left to the reader. Let us consider the quadratic polynomial $f(X)=a X^{2}+b X=X(a X+b)$. Assume that $R$ has an identity; so $a+b=0$ and then $f(X)=a X(X-1)=0$. Putting $X=2$ gives $2 a=0$. The simplest case is when char $R=2$ and $a=1$; that is, $f(X)=X(X+1)$, which is covered in the next example.

Example 3.3. Let $R$ be a ring and $f(X)=X(1+X)$. Now $R$ satisfies $f(X)=0$ if and only if $R$ is Boolean. A ring $R$ is said to be $n$-Boolean ([1]) if 
char $R=2$ and $R$ satisfies $f\left(X_{1}\right) \cdots f\left(X_{n}\right)=0$. So $R$ is 1-Boolean if and only if $R$ is Boolean. A 2-Boolean ring is called a Boolean-like ring $([3,4])$. By Theorem 2.2 if $R$ satisfies $\prod_{i=1}^{n} f\left(X_{i}\right)=0$, then for an $R$-module $M, R(+) M$ satisfies $\prod_{i=1}^{n+1} f\left(X_{i}\right)=0$. Since $\operatorname{char} R(+) M=\operatorname{char} R$, this gives that if $R$ is $n$-Boolean, then $R(+) M$ is $(n+1)$-Boolean. Hence if $R$ is a Boolean ring, then $R(+) M$ is a Boolean-like ring. In [1, Theorem 8$]$ it is shown that $R$ is $n$-Boolean if and only if $R / \operatorname{nil}(R)$ is Boolean, $\operatorname{char} R=2$, and $\operatorname{nil}(R)^{n}=0$. Using this characterization, it is shown in [1, Theorem 9] that $R$ is $n$-Boolean implies $R(+) M$ is $(n+1)$-Boolean. We remark that [1, Theorem 10] says that every Boolean-like ring has the form $R(+) M$ for some Boolean ring $R$ and $R$-module $M$. However, a 3-Boolean ring need not have the form $R(+) M$ where $R$ is 2 -Boolean ([1, page 74]). Note that throughout this example we could replace the polynomial $f(X)=X(1+X)$ by $g(X)=X(1-X)$ since $g(X)=-f(-X)$.

\section{ACKNOWLEDGEMENTS.}

We would like to thank the referee for a number of suggestions for improving the exposition of the paper.

\section{REFERENCES}

[1] D. D. Anderson, Generalizations of Boolean rings, Boolean-like rings and von Neumann regular rings, Comment. Math. Univ. St. Paul. 35 (1986), 69-76.

[2] D. D. Anderson and M. Winders, Idealization of a module, J. Commut. Algebra 1 (2009), 3-56.

[3] A. L. Foster, The idempotent elements of a commutative ring form a Boolean algebra; ring duality and transformation theory, Duke Math. J. 12 (1945), 143-152.

[4] A. L. Foster, The theory of Boolean-like rings, Trans. Amer. Math. Soc. 59 (1946), $166-187$.

M. Bataineh

Department of Mathematics and Statistics

Jordan University of Science and Technology

Irbid 22110

Jordan

E-mail: msbataineh@just.edu.jo

D. D. Anderson

Department of Mathematics

The University of Iowa

Iowa City, IA 52242

USA

E-mail: dan-anderson@uiowa.edu

Received: 9.1.2013.

Revised: 22.7.2013. 